\title{
NANOTECHNOLOGY: A NEW FRONTIER IN VIRUS DETECTION IN CLINICAL PRACTICE
}

\author{
AM Abraham, R Kannangai, *G Sridharan
}

\begin{abstract}
Researchers are expanding the applications of nanotechnology in the field of medicine since mid-2000. These technologies include nanoarrays, protein arrays, nanopore technology, nanoparticles as a contrivance in immunoassays and nanosensors, among others. Nanobiotechnologies are clinically applicable and possess the potential to be useful in laboratory diagnosis of infections in general and viral infections in particular. Nanotechnology is a significant advance in molecular diagnostics. The technology strengthens and expands the DNA and protein microarray methods. In particular, the waveguide technology is an emergent area with many diagnostic applications. Nanosensors are the new contrivance for detection of bioterrorism agents. All these new technologies would have to be evaluated in clinical settings before their full import is appreciated and accepted.
\end{abstract}

Key words: Diagnostics, infectious disease, nanosensors, nanotechnology

The field of medical virology has seen many technological advances in the last three decades in the diagnosis of viral infections. In the 1980s, the enzyme-linked immunosorbent assay (ELISA) was widely introduced in laboratories for the diagnosis of viral infections. The assays could detect antibodies, class-specific immunoglobulins (IgG/ IgM / IgA) to viruses or viral antigens, as custom designed to perform. In the 1990s and the early part of this decade, we saw the rapid development of the polymerase chain reaction (PCR) and its modifications and their application in viral detection in diagnosis of infectious diseases.

In the second half of this decade, we now see a new frontier emerging, and researchers are pushing the envelope to expand the applications of nanotechnology in the field of medicine. The term nanometer refers to a unit which is one billionth of a meter $\left(10^{-9}\right.$ meter $)$. The molecular and sub-molecular technology which is involved in working with devices less than 100 nanometers in size, with its innovative use, is what is broadly called the field of nanotechnology. The science of this area began in the 1980s with the discovery and innovations of a family of unusual hollow carbon molecular aggregations called fullerenes. The molecular structures were biocompatible and did not have any genotoxicity or mutagenicity. The areas of application of nanomolecules include therapeutics, cancer detection, diagnosis and treatment of infectious disease. This editorial will delve into the applications as relevant to medical virology.

*Corresponding author (email: <gopalansridharan@hotmail.com>) Department of Clinical Virology, Christian Medical College, Vellore-632 004, Tamil Nadu, India

Received: 03-05-2008

Accepted: 19-08-2008

\section{Nanotechnology with Application in Infectious Disease Diagnosis}

These technologies include nanoarrays, protein arrays, nanopore technology, nanoparticles (NPs) as a contrivance in immunoassays and nanosensors, among others. Gold NPs and quantum dots (semiconductors) are the most widely used, but new materials are becoming available as more molecular entities are discovered as amenable to nanoscale design and fabrication. Crystal materials like those of gallium, phosphate, quartz, and ceramic are chosen for their durability and piezoelectric properties of developing and retaining an electric potential (charge) when subjected to mechanical stress. Another area of development is nanobiosensors, in which antibody-based piezoelectric nanobiosensors are well developed. ${ }^{[1,2]}$ Molecular technologies will hence advance and be in widespread use within a decade, and the maximum impact will be felt in biomarker research, cancer diagnosis, and detection of infectious microorganisms. Nanotechnology, thus extends the frontiers of molecular diagnostics to the nanoscale.

Nanotechnology-on-a-chip is one more dimension of microfluidic/lab-on-a-chip technology. The analyte detection is quick, sensitive, and has more manipulability when certain NPs are used as tags or labels. Magnetic NP on antibody serves as a label for specific antigen detection in magnetic immunoassay techniques. Here, the magnetic field generated by the magnetically labelled targets is detected with a magnetometer. Gold NP tagged on short segments of DNA (oligos) has been used for detection of genetic sequence in a sample. DNA nanomachines have been documented to function as biomolecular detectors for homogeneous assays. These assays require no separation steps; pipetting, incubation, and measurement steps alone are required. In these homogenous assays, the reaction is in solution format 
without solid phase anchoring, which could interfere with low-affinity interactions. The components of the assay are present in a reaction vessel even at the time of readout.

Yet another significant development is the availability now of sensors for point-of-care viral diagnostics. The technology is an interferometric biosensor immunoassay for direct and label-less detection of viruses. Monochromatic light from a laser source is coupled to a channel waveguide and is guided into four parallel channels (one reference channel and three measuring channels). This facilitates detection simultaneously of three different viruses as the individual channels are coated with specific antibodies. On exiting through the channels, the probe light is interfered, generating an interference pattern (phase change) on a monitor screen. The interference pattern gives information on concentration of virus particles in the reaction channel. ${ }^{[3,4]}$ The detection of avian influenza virus through whole-virus capture on a planar optical waveguide has been described. The assay response is based on the index of refraction changes that occur upon binding of virus particles to unique antigen-specific (haemagglutinin) antibodies on the waveguide surface. ${ }^{[5]}$

Nanobiotechnologies are clinically applicable and possess the potential to be useful in laboratory diagnosis of infections in general and viral infections in particular. Nanotechnology is functional in the design of biochips as they enable the diagnosis at the molecule and single cell level and hence serve as a great advance in molecular diagnostics. Recently, functionalized NPs covalently linked to biological molecules such as antibodies, peptides, proteins, and nucleic acids have been developed as nanoprobes for molecular detection. These functionalized NPs can provide a direct rapid method of detection of viruses with high sensitivity. ${ }^{[6,7]}$

Researchers from the Netherlands have developed a device for fast pathogen detection. It uses a laser coupled to a waveguide composed of four parallel optical channels, each coated with antibodies specific to a certain protein or virus. The application of the technology was demonstrated for the detection of herpes simplex virus type 1 (HSV-1) by coating one of the waveguide channels with the appropriate herpes antibodies. The technique detected the virus over a range of concentrations ranging from as low as $10^{3} / \mathrm{mL}$ to $10^{7} / \mathrm{mL}$. Combining the light exiting from the virus-specific channel and that from a reference channel, an interference pattern is generated. Virus binding to the antibody-coated waveguide is probed by the evanescent field of the guided light modes, causing a phase change and a change in the interference pattern, which is recognized by a monitoring device. The technology is claimed to potentially have wide application, as any antibody can be used to coat a channel for detection. ${ }^{[1]}$ This sensor can be extended to any virus like human immunodeficiency virus (HIV), severe acute respiratory syndrome (SARS) coronavirus, $\mathrm{HBV}, \mathrm{HCV}$, or the avian influenza virus (H5N1), among others. ${ }^{[4]}$

More technological approaches are reported, like application of a silver nanorod array SERS substrate. This is a spectroscopic assay based on surface-enhanced Raman scattering (SERS) using silver nanorod array substrates. The SERS assay can detect spectral differences between viruses, viral strains, and viruses with gene deletions and is a method that can prove useful for rapid diagnostics. ${ }^{[8,9]}$

\section{Applications of Nanotechnology in Virus Detection}

\section{Respiratory viruses}

A microfluidic platform-based detection system is now described. The term refers to precise control and manipulation of fluids contained typically in sub-millimetre scale volume. The assay can detect the avian influenza virus $\mathrm{H} 5 \mathrm{~N} 1$ in throat swab samples by using magnetic forces to manipulate a free droplet containing superparamagnetic particles (ferric oxide-labelled antibody) to concentrate the viruses. The viral RNA is first isolated, purified, and subjected to ultra-fast real-time reverse transcriptase PCR (RT-PCR). This real-time PCR chip is capable of conducting one thermal cycle in 8.5 seconds, which corresponds to 40 cycles of PCR in 5 minutes and 40 seconds. Silicon micromachined into a cantilever and terminated with a disc is the scaffolding for this PCR system. The disc perimeter has the thin film heater and a temperature sensor. A heating rate of $175^{\circ} \mathrm{C} \mathrm{s}^{-1}$ and a cooling rate of $-125^{\circ} \mathrm{C} \mathrm{s}^{-1}$ are achieved due to the system's thermal constant of 0.27 second. A PCR sample encapsulated with mineral oil dispensed onto a glass cover slip placed on the silicon disc is the reaction vessel. The PCR cycle time was 0.5 second by heat transfer from an infrared source through the glass. The real-time PCR is carried out with $100 \mathrm{~nL}$ sample volume using a FAM probe. The system is amenable for testing a large number of samples at one time. ${ }^{[10]}$

The waveguide technology has been used for the successful detection of the avian flu virus in buffer solution. Monoclonal and polyclonal capture antibodies against haemagglutinin proteins were used in the channels. The interference pattern was clearly dose dependent, and the assay had a detection limit as low as $0.0005 \mathrm{HAU} / \mathrm{mL} .{ }^{[1]}$

A method for the respiratory syncytial virus (RSV) consisting of functionalized NPs conjugated to monoclonal antibodies can be used to rapidly and specifically detect $\mathrm{RSV}$ in clinical samples with a great degree of sensitivity. ${ }^{[6]}$

A nanoparticle label technology with highly fluorescent europium (III)-chelate-doped nanoparticle label on highaffinity monoclonal antibodies (anti-hexon) to adenovirus has been tested. The device was highly specific with a detection limit of 5000 virus particles per millilitre of purified virus particles. The sensitivity was improved by three orders of magnitude (800-fold) compared to other standard methods. 
The nanoparticle assay showed low variation in log values and excellent linear relationship to virus concentration. The assay was assessed on nasopharyngeal samples and found to be superior to conventional methods. ${ }^{[12]}$

A separation-free bioaffinity assay technique with the use of dry-chemistry reagents has been developed for influenza virus $\mathrm{A}$ and $\mathrm{B}$ detection at point-of-care setting directly in the respiratory secretion. The new assay method is based on the immunometric assay principle. The immunometric binding reaction takes place on the surface of monodispersed polystyrene microspheres coated with monoclonal antibodies specific for influenza A or B virus antigens. These microspheres work as a solid-phase reaction carrier for the immunocomplex formation and are efficient because they carry thousands of antibody molecules with multivalent binding properties. Fluorescence from the surface of the individual microspheres is measured directly from the reaction mixture without any separation using a fluorometer. ${ }^{[13]}$

Quantum dots (QD) are fluorescent semiconducting nanocrystals which have broad excitation spectra and narrow emission spectra and good stability on exposure to light. ${ }^{[14]}$ These are good labels and have successfully been used to study RSV pathogenesis using antibody or oligoRNA probes. Research is now ongoing to develop QD mixes to simultaneously detect multiple respiratory viruses. ${ }^{[1,2]}$

Additional emergent technologies include the triangulation identification for genetic evaluation of risk (TIGER), which uses high performance electro-spray mass spectrometry to identify the base composition of PCR products and the matrix-assisted laser desorption-ionizationtime of flight-mass spectrometry (MALDI-TOF), which generates information on PCR product size and composition based on mass charge ratios. Rapid RT-PCR followed by ESI-MS (electrospray ionization-mass spectrometry) analysis has been used to simultaneously identify all species of influenza viruses with clade-level resolution, identify mixed viral populations, and monitor global spread and emergence of novel viral genotypes. This high-throughput method promises to become an integral component of influenza surveillance. ${ }^{[15]}$

\section{Human immunodeficiency virus (HIV)}

A new nanoparticle-based biobarcode amplification (BCA) assay has been developed for early and sensitive detection of HIV-1 capsid (p24) antigen. Here, anti-p24 antibody-coated microplates capture viral antigen (p24) and are linked to a detection monoclonal antibody with avidin label as the detection probe. This immune complex is detected by streptavidin-coated nanoparticle-based biobarcode DNAs for signal amplification. The signal detection uses a chip-based scanometric method. The modified BCA assay exhibited detection as low as 0.1 $\mathrm{pg} / \mathrm{mL}$ and was approximately 150-fold more sensitive than conventional ELISA. In addition, the BCA assay detected HIV-1 infection three days earlier than ELISA in seroconversion samples and hence may serve as an alternate testing strategy to HIV RNA detection. ${ }^{[7]}$

Hepatitis $B$ virus $(H B V)$, hepatitis $C$ virus $(H C V)$, and hepatitis $E$ virus (HEV)

A visual gene-detecting technique using gold nanoparticle-labelled gene probes has been described. Nanoparticle-supported 3'-end-mercapto-derivatized oligonucleotide was used as the detection probe, and 5'end-amino-derivatized oligonucleotide immobilized on glass surface was used as capturing probe. The target DNA was detected visually by sandwich hybridization using the highly sensitive "nano-amplification" and silver staining. The HBV, HCV, and HBV/HCV gene chips with gold/silver NP staining amplification method were shown to be useful in detecting these viruses in patients' samples. The detection readout was the resonance Rayleigh light scattering (RLS) spectroscopy to monitor the immobilization of gene probes on gold nanoparticle surfaces. ${ }^{[16]}$

Simultaneous detection of antibodies to HBV and HCV components has been reported using a protein chip assay with nano-gold immunological amplification and silver staining method. Glass slides are used as solid supports (protein chip), on which several antigens of HBV (HBsAg, $\mathrm{HBeAg}$, and $\mathrm{HBcAg}$ ) and $\mathrm{HCV}$ (a mixture of NS3, NS5, and core antigens) were immobilized. In this assay, colloidal nano-gold-labelled staphylococcal protein A (SPA) was used as an indicator. The immunogold silver staining enhancement technique was applied to amplify the detection signals. The product was a black image on an array spot that was visible to the naked eyes. The assay could detect specific antibody as low as $3 \mathrm{ng} / \mathrm{mL}$. ${ }^{[17]}$

An array-based nano-amplification technique method for the detection of HEV has been developed by utilizing nanogold-labelled oligonucleotide probes coupled with silver stain enhancement and the microarray technique. The 5'end- $\mathrm{NH}_{2}$ modified oligonucleotide probes are immobilized on the DNA chip base as the capture probe. The detection probe consists of the 3'-end-SH modified oligonucleotide probe and nano-gold colloid. The HEV virus RNA was amplified by the RT-PCR/PCR one-step amplification, and the cDNA was hybridized with the capture probes and the detection probes on microarray. The signal was amplified by silver stain enhancement identified by the unaided eye. The microarray was shown to detect $100 \mathrm{fM}$ of amplicon with the image development time as short as 2 minutes. A similar technique is also described for HAV. ${ }^{[18,19]}$

Herpes simplex virus (HSV)

The Young interferometer sensor has been applied 
for direct detection of viruses. The technology has been validated for HSV-1 but is broadly applicable. Detection of HSV-1 virus particles is achieved by exposing the viruscontaining sample to a sensor surface coated with a specific antibody against HSV-1. The Young interferometer sensor was shown to detect HSV-1 at very low concentrations (850 particles $/ \mathrm{mL}$ ) and even directly in serum samples. ${ }^{[1]}$

Isothermal DNA amplification through EXPAR (exponential amplification reaction) coupled with gold nanosphere-based visual colorimetric readout facilitated by aggregation of DNA-functionalized gold nanospheres has been developed for HSV detection. EXPAR amplifies short oligonucleotide trigger sequence $10^{6}$ - to $10^{9}$-fold in $<10$ minutes at $55^{\circ} \mathrm{C}$. This allows for the activity and stability of the polymerase and nicking endonuclease required for the reaction..$^{[1]}$

\section{Other viral infections}

A novel signal amplification technology for a human papillomavirus (HPV)-DNA hybridization assay based on fluorescein diacetate (FDA) nanocrystals has been developed. The FDA nanocrystals are nearly water-insoluble precursors of fluorescein, which is dissolved and hydrolyzed by treatment with an organic solvent/hydroxide mixture. This is treated with a polymeric surfactant to create a stable, nanosized colloid with an interface for coupling streptavidin molecules. Initially, biotin labels incorporated in primers result in PCR products for the quantitative detection of the biotinylated HPV-specific DNA products, amplified in a standard PCR procedure. The amplified HPV-DNA, labelled with biotin, was hybridized with the immobilized probes of HPV 16, HPV 18, or HPV 45 in 96-well microplates. After the affinity reaction with the streptavidin FDA nanoprobe, the FDA molecules were dissolved and concomitantly converted into fluorescein by the special reagent (DMSO and $1 \mathrm{M} \mathrm{NaOH}$ in a 1:1 ratio). Florescence emission in the microwells of the plate is excited at $485 \mathrm{~nm}$ and recorded at $538 \mathrm{~nm}$ in an fmax fluorescence microplate reader. This approach resulted in high selectivity, short incubation times, and high sensitivity. This innovative method allows rapid detection of small amounts of target sequence in a fewer number of PCR cycles. ${ }^{[14]}$

Diarrhoea-causing viruses today are a major public health concern, and newer agents with potential for large food-borne outbreaks have been described. Norovirus is a leading cause of gastroenteritis in many parts of the world. A matrix-assisted laser desorption ionization and nanospray mass spectrometry was developed and evaluated for norovirus detection using different approaches. The detection of norovirus capsid protein was examined by using mass spectrometry-based methods: scanning of intact proteins, peptide mass fingerprinting, and peptide sequencing. Detection of intact target protein was found to be an unsatisfactory approach. Detection of peptides by peptide mass fingerprinting (16 peptides) and detection of the $56-\mathrm{kDa}$ norovirus capsid protein as norovirus viruslike particles (VLPs) in spiked human stool samples after extraction were tested. The peptide mass fingerprinting was found to fail. Peptide sequencing using nanospray tandem MS was the best method for identification of $250 \mathrm{fM}$ or more of capsid protein in stool extracts. ${ }^{[20]}$

Microscale and nanoscale cantilever beams have been used as highly sensitive mass detectors. Antibodies can be efficiently bound on cantilevers. The binding of a probe to an analyte results in deflection of the cantilever, and this can be detected by a detection unit. A counterbalancing force required to restore the cantilever to its original position may be magnetic or electrical. Scaling down the area of the cantilever allows a decrease in minimum detectable mass limit, while scaling down the thickness enables the resonant frequencies to be within the measurable range. Fabricated arrays of silicon cantilever beams as nanomechanical resonant sensors to detect the mass of individual virus particles have been developed. The cantilever beams are in the range of 4-5 $\mu \mathrm{m}$ in length, $1-2 \mu \mathrm{m}$ in width, and 20$30 \mathrm{~nm}$ in thickness by attachment of protein layers. The frequency spectra of the cantilever beams are measured using a laser doppler vibrometer. The virus particle binding on the cantilever beam surface changes the resonant frequency which is measured. This technology has been successfully demonstrated for the detection of a single vaccinia virus particle (average mass of $9.5 \mathrm{fg}$ ). Specific capture of the antigens requires attachment of antibodies, which can be in the same range of thickness as these cantilever sensors. These devices can be very useful as components of biosensors for the detection of air-borne virus particles.

The biosensing method most explored in virus detection is the SERS due to its ability to detect even a single virus particle. Recently it has been shown that a silver nanorod array fabricated using oblique-angle deposition (OAD) acts as an extremely sensitive SERS substrate. The OAD is a simple physical-vapour-deposition technique where the vapour arrives at close to the grazing angle. This process ensures preferential growth of nanorods on the substrate in the direction of deposition due to the shadow casting effect.

The Raman spectra of viruses can be used to rapidly and readily distinguish molecular fingerprints for classifying and identifying viruses. The SERS technique is highly promising for designing new virus-detection schemes. The spectra can be collected within 1 minute at a virus volume less than 5 $\mu \mathrm{L}$. SERS establishes reproducible molecular fingerprints of several important human viruses and can be an ideal screen for bioterrorism agents.

\section{Summary}

The use of nanoparticles as tags or labels allows for 
the detection of infectious agents in small sample volumes directly in a very sensitive, specific, and rapid format at lower costs than current in-use technologies. This advance in early detection enables accurate and prompt treatment. The quantum dot technology is currently the most widely employed nanotechnology. The recently emerging cantilever technology is the most promising. The technology strengthens and expands the DNA and protein microarray methods and has applications in genomic analysis, proteomics, and molecular diagnostics. Waveguide technology is an emergent area with many diagnostic applications. Nanosensors are the new contrivance for detection of bioterrorism agents. All these new technologies would have to be evaluated in clinical settings before their full import is appreciated and accepted.

\section{References}

1. Jain KK. Nanotechnology in clinical laboratory diagnostics. Clin Chim Acta 2005;358:37-54

2. Demidov VV. Nanobiosensors and molecular diagnostics: A promising partnership. Exp Rev Mol Diagn 2004;4:267-8.

3. Ymeti A, Greve J, Lambeck PV, Wink T, van Hövell SW, Beumer TA, et al. Fast, ultrasensitive virus detection using a young interferometer sensor. Nano Lett 2007;7:394-7.

4. Ymeti A, Kanger JS, Greve J, Besselink GA, Lambeck PV, Wijn R, et al. Integration of microfluidics with a four-channel integrated optical Young interferometer immunosensor. Biosens Bioelectron 2005;20:1417-21.

5. Xu J, Suarez D, Gottfried DS. Detection of avian influenza virus using an interferometric biosensor. Anal Bioanal Chem 2007;389:1193-9.

6. Tripp RA, Alvarez R, Anderson B, Jones L, Weeks C, Chen W. Bioconjugated nanoparticle detection of respiratory syncytial virus infection. Int J Nanomed 2007;2:117-24.

7. Tang S, Zhao J, Storhoff JJ, Norris PJ, Little RF, Yarchoan $\mathrm{R}$, et al. Nanoparticle-Based biobarcode amplification assay (BCA) for sensitive and early detection of human immunodeficiency type 1 capsid (p24) antigen. J Acquir Immune Defic Syndr 2007;46:231-7.

8. Shanmukh S, Jones L, Driskell J, Zhao Y, Dluhy R, Tripp RA. Rapid and sensitive detection of respiratory virus molecular signatures using a silver nanorod array SERS substrate. Nano Lett 2006;6;2630-6.

9. Zhao Y, Shanmukh S, Liu Y, Jones L, Dluhy RA, Tripp
RA. Silver nanorod arrays can distinguish virus strains. Nanotechnology. Available from: http \www.spie.org. [last accessed on 2008 May 2].

10. Neuzil P, Zhang C, Pipper J, Oh S, Zhuo L. Ultra fast miniaturized real-time PCR: 40 cycles in less than six minutes. Nucl Acids Res 2006;34:e77.

11. Pipper J, Inoue $\mathrm{M}, \mathrm{Ng}$ LF, Neuzil P, Zhang Y, Novak L. Catching bird flu in a droplet. Nat Med 2007;13:1259-63.

12. Valanne A, Huopalahti S, Soukka T, Vainionpää R, Lövgren T, Härmä H. A sensitive adenovirus immunoassay as a model for using nanoparticle label technology in virus diagnostics. J Clin Virol 2005;33:217-23.

13. Koskinen JO, Vainionpää R, Meltola NJ, Soukka J, Hänninen PE, Soini AE. Rapid method for detection of influenza A and $\mathrm{B}$ virus antigens by use of a two-photon excitation assay technique and dry-chemistry reagents. J Clin Microbiol 2007;45:3581-8.

14. Chan CP, Tzang LC, Sin K, Ji S, Cheung K, Tam T, et al. Biofunctional organic nanocrystals for quantitative detection of pathogen deoxyribonucleic acid. Anal Chim Acta 2007;584:7-11.

15. Sampath R. Global surveillance of emerging influenza virus genotype by mass spectrometry. PLoS One 2007;2:2489.

16. Wang YF, Pang DW, Zhang ZL, Zheng HZ, Cao JP, Shen JT. Visual gene diagnosis of HBV and HCV based on nanoparticle probe amplification and silver staining enhancement. J Med Virol 2003;70:205-11.

17. Duan L, Wang Y, Li SS, Wan Z, Zhai J. Rapid and simultaneous detection of human hepatitis $B$ virus and hepatitis $\mathrm{C}$ virus antibodies based on a protein chip assay using nano-gold immunological amplification and silver staining method. BMC Infect Dis 2005;5:53.

18. Wan Z, Wang Y, Li SS, Duan L, Zhai J. Development of arraybased technology for detection of HAV using Gold-DNA probes. J Biochem Mole Biol 2005;38:399-406.

19. Liu HH, Cao X, Yang Y, Liu MG, Wang YF. Array-based nano-amplification technique was applied in detection of hepatitis E virus. J Biochem Mol Biol 2006;39:247-52.

20. Colquhoun DR, Schwab KJ, Cole RN, Halden RU. Detection of norovirus capsid protein in authentic standards and in stool extracts by matrix-assisted laser desorption ionization and nanospray mass spectrometry. Appl Environ Microbiol 2006;72:2749-55.

Source of Support: Nil, Conflict of Interest: None declared. 